\title{
circRNA-0006896-miR1264-DNMT1 axis plays an important role in carotid plaque destabilization by regulating the behavior of endothelial cells in atherosclerosis
}

\author{
YAN WEN ${ }^{1,2}$, YAO CHUN $^{2}$, ZHONG QING LIAN $^{2}$, ZHANG WEI YONG ${ }^{2}$, YANG MEI LAN ${ }^{2}$, LIAO HUAN $^{2}$, \\ CHEN YI XI $^{2}$, LI SHU JUAN ${ }^{2}$, ZHONG WEN QING ${ }^{2}$, CHENG JIA $^{2}$ and ZHANG HUAN JI ${ }^{3}$ \\ ${ }^{1}$ General Practice Department, The Eighth Affiliated Hospital of Sun Yat-Sen University; \\ ${ }^{2}$ Office of Scientific Research and Development, Sun Yat-Sen University; ${ }^{3}$ Cardiovascular Department, \\ The Eighth Affiliated Hospital of Sun Yat-Sen University, Shenzhen, Guangdong 518000, P.R. China
}

Received March 12, 2020; Accepted October 27, 2020

DOI: $10.3892 / \mathrm{mmr} .2021 .11950$

\begin{abstract}
Atherosclerosis (AS) is a chronic inflammatory disease of the vascular wall with multiple causes. AS is the primary pathological basis of cardiovascular disease and stroke. Moreover, carotid plaque rupture and thrombus formation are the main causes of ischemic stroke. Therefore, understanding the formation of carotid plaques may help improve the prediction and prevention of cardiovascular and cerebrovascular events. Endothelial cell dysfunction results in re-endothelialization and angiogenesis in atherosclerotic plaques, thus promoting plaque destabilization. The aim of the present study was to evaluate the effect of circular RNA (circRNA) molecules in serum exosomes (serum-Exos) from patients with stable plaque atherosclerosis (SA) and unstable/vulnerable plaque atherosclerosis (UA). Specifically, the effect of circRNA on human umbilical vein endothelial cell (HUVEC) behavior and the mechanisms underlying plaque destabilization in AS were evaluated. Serum-Exos were isolated, then identified using transmission electron microscopy, nanoparticle tracking analysis and western blotting. The serum-Exo-circRNA expression profile of patients with SA or UA was investigated using a circRNA array. The relationship between circRNA-006896 in serum-Exos and biochemical parameters of patients with SA and UA were analyzed using Spearman's correlation. In addition, HUVECs were incubated with serum-Exos for in vitro functional assays. The present study demonstrated that circRNAs expression profiles in SA and UA serum-Exos were significantly different, indicating a potential role for
\end{abstract}

Correspondence to: Dr Zhang Huan Ji, Cardiovascular Department, The Eighth Affiliated Hospital of Sun Yat-Sen University, 3025 Shennan Middle Road, Futian, Shenzhen, Guangdong 518000, P.R. China

E-mail: huanji13688808979@163.com

Key words: atherosclerosis, human umbilical vein endothelial cell, serum exosomes, circular RNA-0006896, microRNA1264
circRNAs in carotid plaque destabilization. The expression of circRNA-0006896 was positively correlated with triglyceride, low-density lipoprotein cholesterol (LDL-C) and C-reactive protein levels, and negatively correlated with albumin levels in patients with UA. However, circRNA-0006896 expression was positively correlated with LDL-C in patients with SA. Using bioinformatic analysis, a competing endogenous RNA (ceRNA) network was selected to study the regulatory roles of circRNA-0006896 in serum-Exos. Additionally, in HUVECs treated with serum-Exos derived from patients with UA, the expression of circRNA-0006896 in HUVECs was upregulated. This was accompanied by decreased expression of microRNA-1264 and SOCS3, increased levels of DNMT1 and phosphorylated STAT3. HUVEC proliferation and migration were significantly increased in the UA group, compared with the mock and SA groups. This finding indicates that the circRNA-0006896-miR-1264-DNMT1 axis plays an important role in carotid plaque destabilization by regulating the behavior of endothelial cells. Moreover, it suggests that circRNA-0006896 may represent a therapeutic target for controlling JNK/STAT3 signaling in HUVECs. Thus, this study may provide insight on potential interventions against vulnerable plaque formation in patients with AS.

\section{Introduction}

Atherosclerosis (AS) is a chronic inflammatory disease of the vascular wall with multiple causes. It is characterized by the deposition of lipids in the arterial wall, infiltration of immune cells, and formation of fibrous caps (1). AS is the leading pathological cause of cardiovascular disease and stroke. Carotid plaque rupture and thrombus formation are the main causes of ischemic stroke. Patients with unstable/vulnerable plaque AS (UA) are prone to rupture and thrombus formation, leading to cardiovascular or cerebrovascular events, such as acute coronary syndrome, ischemic stroke, and peripheral vascular disease (2). Therefore, identifying the properties of carotid plaques may help improve the prediction and prevention of cardiovascular and cerebrovascular events. Previous reports noted that several factors sampled from plasma or serum may 
contribute to carotid plaque vulnerability, symptom status, degree of stenosis and stroke risk. These factors include the serum protein C-X-C motif chemokine ligand 9, microRNA (miR)-17-92, and circular RNA (circRNA)-089763 (3-5).

Exosomes are extracellular vesicles secreted by living cells. They can circulate in biological fluids, permeate biological barriers (for example, the brain-blood barrier or the placental barrier), target recipient cells, and thus share intercellular signaling molecules between cells $(6,7)$. Exosomes may represent a potential and effective tool for the diagnosis and treatment of diseases, due to their extensive presence in the body and relative ease of sampling. Exosomes can also be a source of disease-related proteins, miRNAs, and circRNAs (8-10). Transfer of exosome-enclosed proteins, miRNAs and circRNAs has been identified as an intercellular communication method in cardiovascular diseases, such as atherosclerosis $(11,12)$. The signaling molecules carried by exosomes circulate in the body and can affect the biological behavior of target cells, thereby acting as key factors in disease development.

circRNAs are endogenous non-coding RNA molecules that differ from linear RNA by their structure, a closed covalent loop lacking $3^{\prime}$ or 5' polarity (13). circRNAs can sponge miRNAs through stable complementary binding and regulate gene expression. Numerous studies have demonstrated that circRNAs are stable and abundant within exosomes; moreover, they may be key to the mechanisms through which exosomes promote certain disease $(1,14,15)$.

Emerging evidence suggests that endothelial cell dysfunction induces an increase in cell proliferation and migration that leads to re-endothelialization and angiogenesis of carotid plaques, which in turn promotes plaque destabilization, plaque rupture, and thrombus formation (16-18). However, whether exosomal circRNAs in the serum of patients with carotid plaques are involved in this process remains unknown.

The aim of the present study was to determine whether exosomal circRNAs were associated with UA formation. Specifically, the regulatory role of serum exosomal circRNAs on endothelial cell behavior was examined in patients with $\mathrm{UA}$, in order to identify novel mechanisms underlying plaque stability.

\section{Materials and methods}

Patient recruitment. The present study was approved by The Ethics Committee of The Eighth Affiliated Hospital of Sun Yat-Sen University. Written informed consent was obtained from all participants or their families. A total of 42 patients diagnosed with carotid plaque under B-ultrasound examination at The Eighth Affiliated Hospital of Sun Yat-Sen University from March 2017 and December 2017 were enrolled in this study. The exclusion criteria were: i) total occlusion lesion; ii) acute coronary syndrome; iii) cardiac shock; and iv) presence of cardiomyopathy, severe anemia, severe renal impairment, or malignant tumor.

Carotid plaque images were obtained using a 1.5-T magnetic resonance system (1.5-T system; Philips Healthcare). A volume isotropic turbo spin echo acquisition (VISTA) sequence was used. The iMap parameters were as follows: i) T1-weighted VISTA, Time of Repetition (TR)/Time of Echo
$(\mathrm{TE})=400 / 16 \mathrm{~ms}$, refocusing angle $=60^{\circ}$, thickness $=1 \mathrm{~mm}$, field of view $=18 \mathrm{~cm}$, matrix $=384 \times 384$, SENSE factor $=2$, number of signal averaging=2); ii) T2-weighted VISTA, $\mathrm{TR} / \mathrm{TE}=3500 / 119 \mathrm{~ms}$, refocusing angle $=60^{\circ}$, thickness $=1 \mathrm{~mm}$, field of view $=18 \mathrm{~cm}$, matrix $=384 \times 384$, SENSE factor $=2$, number of signal averaging $=2$, and iii) time of flight Magnetic Resonance Angiography, TR/TE $=16 / 6.9 \mathrm{~ms}$, flip angle $=60^{\circ}$, thickness $=1.5 \mathrm{~mm}$, field of view $=22 \mathrm{~cm}$, matrix $=512 \times 512$, SENSE factor $=1.8$, number of signal averaging $=2$. Patients with lipid-rich and necrotic core plaques were diagnosed with UA ( $\mathrm{n}=20)$ according to previous studies (signal intensity ratio $\geq 1.25$ using T1-weighted VISTA) $(19,20)$. All other patients were diagnosed with SA $(n=22)$. Clinical and demographic characteristics, as well as information on ongoing treatments are presented in Table I.

Exosome isolation. A volume of $100 \mu 1$ serum was collected from every patient. All samples were combined into a UA serum pool and an SA serum pool. Both serum pools were centrifuged for $20 \mathrm{~min}$ at $2,000 \mathrm{x} \mathrm{g}, 30 \mathrm{~min}$ at $10,000 \mathrm{x} \mathrm{g}$, then $20 \mathrm{~min}$ at $14,000 \mathrm{x} \mathrm{g}$. All centrifugation steps were performed at $4^{\circ} \mathrm{C}$. Exosomes were then isolated using a GET-Exosome isolation kit (GenExosome Technologies, Inc.) according to the manufacturer's instructions. The concentration of exosomes was evaluated using a BCA assay. All serum-Exos were stored at $-80^{\circ} \mathrm{C}$ or immediately used for further experiments.

circRNA isolation. Exosomal circRNAs were extracted from serum-Exos using TRIzol ${ }^{\circledR}$ (Thermo Fisher Scientific, Inc.). For digestion of linear RNAs, $1 \mathrm{mg}$ of total RNA was incubated with $2 \mathrm{U} / \mu \mathrm{g}$ RNase R for $1 \mathrm{~h}$ at $37^{\circ} \mathrm{C}$ (Thermo Fisher Scientific, Inc.). The purity and concentration of the RNA samples were assessed with a NanoDrop ${ }^{\mathrm{TM}}$ spectrophotometer (NanoDrop ${ }^{\mathrm{TM}}$ Technologies; Thermo Fisher Scientific, Inc.).

circRNA microarray analysis. Following extraction from SA or UA serum-Exos pools, circRNAs were divided into three parallel samples for each group (SA-Exos-1, 2 and 3 vs. UA-Exos-1, 2 and 3) and used for circRNA microarray analysis. The Human CircRNA Array v2 (CapitalBio Technology Co., Ltd.) was used; with each slide containing four identical arrays (4x180K format), representing $\sim 170,340$ human circRNAs. A total of 4,974 Agilent control probes were included in the array, and 7,775 circRNAs were examined. GeneSpring software (version 13.0; Agilent Technologies, Inc.) was used to analyze, standardize and control the quality of the circRNA microarray data. To identify the differentially expressed genes, a fold change threshold of $\geq 1.2$ or $\leq-1.2$ was used. Statistical analysis was carried out using Student's t-test, with $\mathrm{P}<0.05$ indicating a statistically significant difference. Cluster 3.0 (Stanford University) was used for $\log _{2}$ transformation and median centering of the data, which were further analyzed through hierarchical clustering using the average linkage criterion. The miRanda (http://www. miranda.org/) and CircInteractome (https://circinteractome. nia.nih.gov/) tools were used to select the target miRNAs of circRNA-0006896 and construct a circRNA-miRNA network. A competing endogenous RNA (ceRNA) network map was constructed using Cytoscape. 
Table I. Baseline clinical and biochemical characteristics of the enrolled patients.

\begin{tabular}{|c|c|c|c|}
\hline Variables & $\mathrm{SA}(\mathrm{n}=22)$ & $\mathrm{UA}(\mathrm{n}=20)$ & P-value \\
\hline Age, years, mean (SD) & $47.8(11.08)$ & $53.1(5.84)$ & 0.072 \\
\hline Sex, male, n (\%) & $9(81.1)$ & $7(70)$ & 0.654 \\
\hline Smoking, n (\%) & $9(81.8)$ & $6(60)$ & 0.507 \\
\hline Asymptomatic, n (\%) & $2(18.2)$ & $4(40)$ & 0.426 \\
\hline Amaurosis fugax, n (\%) & $5(45.5)$ & $4(40)$ & 0.863 \\
\hline Transient ischemic attack, $\mathrm{n}(\%)$ & $4(36.4)$ & $2(20)$ & 0.557 \\
\hline SBP, mmHg, mean (SD) & $120.7(13.81)$ & $130.80(22.29)$ & 0.314 \\
\hline DBP, mmHg, mean (SD) & $76.43(10.22)$ & $84.60(18.40)$ & 0.085 \\
\hline TC, mmol/1, mean (SD) & $5.32(1.46)$ & $4.74(1.22)$ & 0.314 \\
\hline TG, mmol/l, median (IQR) & $1.47(0.9-1.6)$ & $1.62(1.1-1.9)$ & 0.251 \\
\hline HDL-C, mmol/l, median (IQR) & $1.03(1.0-1.1)$ & $1.01(0.9-1.1)$ & 0.918 \\
\hline LDL-C, mmol/1, mean (SD) & $2.84(0.15)$ & $3.33(0.18)$ & 0.035 \\
\hline GLU, mmol/l, median (IQR) & $5.49(5.1-6.4)$ & $5.61(5.6-6.0)$ & 0.468 \\
\hline $\mathrm{GHb}, \%$, median (IQR) & $5.70(5.5-5.8)$ & $5.75(5.5-6.1)$ & 0.842 \\
\hline Uric acid, $\mu \mathrm{mol} / \mathrm{l}$, median (IQR) & $377(324.0-408.0)$ & $447(363.0-536.0)$ & 0.261 \\
\hline ALT, U/1, median (IQR) & $38.00(26.8-42.8)$ & $31.50(18.8-55.5)$ & 0.705 \\
\hline ALB, U/1, median (IQR) & $42.60(40.6-43.2)$ & $39.75(37.1-42.0)$ & 0.085 \\
\hline AST, U/1, median (IQR) & $30.00(24.6-40.4)$ & $25.95(18.2-53.7)$ & 0.387 \\
\hline BUN, mmol/l, median (IQR) & $4.60(3.7-4.8)$ & $6.08(5.0-7.5)$ & 0.085 \\
\hline GGT, U/1, median (IQR) & $30.60(22.2-50.4)$ & $38.80(24.9-52.4)$ & 0.654 \\
\hline $\mathrm{sCr}, \mu \mathrm{mol} / \mathrm{l}$, median (IQR) & $71.36(50.0-143.0)$ & $77.49(55-153.0)$ & 0.332 \\
\hline CRP, mg/l, mean (IQR) & $10.55(1.42-33.77)$ & $17.95(3.56-40.68)$ & 0.029 \\
\hline
\end{tabular}

SBP, systolic blood pressure; DBP, diastolic blood pressure; TC, total cholesterol; TG, triglyceride; HDL-C, high-density lipoprotein cholesterol; LDL-C, low-density lipoprotein cholesterol; GLU, glucose levels upon admission; GHb, glycated hemoglobin; ALT, alanine aminotransferase; ALB, serum albumin; AST, aspartate aminotransferase; BUN, blood urea nitrogen; GGT, $\gamma$-glutamyl transpeptidase; sCr, serum creatinine; $\mathrm{CRP}$, C-reactive protein; SA, stable plaque atherosclerosis; UA, unstable/vulnerable plaque atherosclerosis; IQR, interquartile range.

Cell culture. Human umbilical vein endothelial cells (HUVECs) were purchased from the American Type Culture Collection and cultured in RPMI-1640 medium supplemented with $10 \%$ FBS (both from Thermo Fisher Scientific, Inc.) at $37^{\circ} \mathrm{C}$ in a humidified atmosphere containing $5 \% \mathrm{CO}_{2}$. At $80-90 \%$ confluence, cells were sub-cultured at a dilution ratio of 1:3. All experiments were performed with cells in the logarithmic growth phase. For experiments, the cells were cultured to approximately $80 \%$ confluence prior to incubation with serum exosomes from AS patients with UA (UA-Exos) or stable plaque (SA-Exos).

Exosome tracking. Isolated exosomes were labelled using a GET-Exosome labeling kit (GenExosome Technologies, Inc.) according to the manufacturer's protocol. Briefly, $500 \mu \mathrm{g} / \mathrm{ml}$ serum-Exos (or PBS as a control) were mixed with an equal volume of GenExosome Exo ${ }^{\mathrm{PKH} 26}$ tracking dye for $10 \mathrm{~min}$ at $37^{\circ} \mathrm{C}$. The free dye was then removed using GenExosome centrifugal columns (10-kDa cutoff). Next, $1 \times 10^{5}$ cell/ml HUVECs were seeded in a $35-\mathrm{mm}$ confocal dish and incubated with $50 \mu \mathrm{l} 500 \mu \mathrm{g} / \mathrm{ml}$ labelled UA-Exos or SA-Exos (or PBS as a control) in RPMI-1640 medium supplemented with $1 \%$ exosome-free $\mathrm{FBS}$ at $37^{\circ} \mathrm{C}$ for $24 \mathrm{~h}$. Internalized, labelled exosome signals were visualized under a confocal fluorescence microscope (magnification, x400; Leica Microsystems, Inc.).
Dual-luciferase reporter assay. Dual-luciferase reporter assays were performed using the psiCHECK2 vector (Promega Corporation). First, the wild-type (WT) or mutant (MUT) circRNA-0006896 sequences of the putative binding-site for miR1264 were subcloned into the psiCHECK2 vector. Next, 293 cells (ATCC) were seeded in 24-well plates at a density of $5 \times 10^{4}$ cells/well and co-transfected with of psiCHECK2-circRNA-0006896-WT or -MUT, together with the miR-1264 mimic or mimic-miR-control (0.2 mg luciferase plasmid and $50 \mathrm{nM}$ miRNA mimic) using Lipofectamine ${ }^{\circledR}$ 3000 (Invitrogen, Thermo Fisher Scientific, Inc.). The cells were harvested $48 \mathrm{~h}$ after transfection, and luciferase activity was assessed with a Dual-Luciferase ${ }^{\circledR}$ Reporter Assay System (Promega Corporation). Results are presented as relative Renilla luciferase activities, which were normalized to firefly luciferase activities.

RNA fluorescence in situ hybridization (FISH). RNA FISH was performed to determine the location of circRNA-0006896 and miR1264 in HUVECs. Biotin-labelled circRNA0006896 (5'-biotin-TGTATG GGG AGA TGT CTCTCTTTGAGTTAGGTCTAAAGATGATGG-3') and digoxigenin-labeled miR-1264 probes (5'-digoxigeninAACAGGTGCTCAAATAAGACTTG 3') were designed and synthesized by Guangzhou RiboBio Co., Ltd. HUVECs 
were seeded in 6-well plates at a density of $1 \times 10^{5}$ cells $/ \mathrm{ml}$ and incubated with $200 \mu \mathrm{l}$ UA-Exos $(500 \mu \mathrm{g} / \mathrm{ml})$ or SA-Exos $(500 \mu \mathrm{g} / \mathrm{ml})$ or PBS (mock group) in $2 \mathrm{ml}$ conditioned medium at $37^{\circ} \mathrm{C}$ for $24 \mathrm{~h}$. After immobilization with $4 \%$ paraformaldehyde, cells were prehybridized in prehybridization buffer (1X PBS with $0.5 \%$ Triton X-100), then hybridized in hybridization buffer (40\% formamide; $10 \%$ Dextran sulfate; 1X Denhardt's solution; 4X saline sodium citrate buffer; $10 \mathrm{mM}$ DDT, $1 \mathrm{mg} / \mathrm{ml}$ yeast transfer RNA; $1 \mathrm{mg} / \mathrm{ml}$ sheared salmon sperm DNA) with $20 \mathrm{nmol}$ biotin-labelled probes specific for circRNA-0006896 and $20 \mathrm{nmol}$ digoxigenin-labelled locked nucleic acid miR-1264 probes at $60^{\circ} \mathrm{C}$ overnight. The signals of biotin-labelled probes were detected using Cy3-Streptavidin (Thermo Fisher Scientific, Inc.). The signals of digoxigenin-labelled locked nucleic acid miR-1264 probes were detected using the Molecular Probes ${ }^{\mathrm{TM}} \mathrm{TSA}^{\mathrm{TM}}$ kit with Alexa Fluor ${ }^{\mathrm{TM}} 488$ tyramide reagent (Thermo Fisher Scientific, Inc.). Finally, the cells were incubated with 4',6-diamidino-2-phenylindole (DAPI; 1:100, Thermo Fisher Scientific, Inc.) for $15 \mathrm{~min}$ at room temperature. The images were acquired using a confocal microscope (magnification, x600; Leica Microsystems, Inc.).

$R N A$ extraction and reverse-transcription quantitative $(R T-q)$ $P C R$. Total RNAs were extracted from pooled serum-Exos samples, serum-Exos samples from individual patients or HUVECs by using TRIzol ${ }^{\circledR}$ reagent (Thermo Fisher Scientific, Inc.). The individual serum-Exos samples was isolated from a $500-\mu 1$ volume of individual patient serum using a GET-Exosome isolation kit (GenExosome Technologies, Inc.). circRNAs or DNMT1mRNA were reverse transcribed to cDNA with a RevertAid ${ }^{\mathrm{TM}} \mathrm{H}$ Minus First-Strand cDNA Synthesis Kit (Fermentas, Inc.). The reverse-transcription reaction was carried out for $60 \mathrm{~min}$ at $42^{\circ} \mathrm{C}$, followed by a second step of $10 \mathrm{~min}$ at $70^{\circ} \mathrm{C}$ and a final hold at $4^{\circ} \mathrm{C}$. miR-1264 was reverse transcribed to cDNA with a Mir-X ${ }^{\mathrm{TM}}$ miRNA First-Strand Synthesis Kit (Clontech Laboratories, Inc.). The RT reaction was carried out for $60 \mathrm{~min}$ at $37^{\circ} \mathrm{C}$, followed by a second step of $5 \mathrm{~min}$ at $85^{\circ} \mathrm{C}$ and a final hold at $4^{\circ} \mathrm{C}$. All cDNA was synthesized from $1 \mu \mathrm{g}$ of total RNA. The qPCR was carried out using a SYBR ${ }^{\mathrm{TM}}$ Green PCR Master Mix Kit (Thermo Fisher Scientific, Inc.).

The sequences of the primers (all from GeneCopoeia) used were as follows: i) circRNA-0006896 forward, 5'-TTGGGA AGCCTGGAATATGA-3' and reverse, 5'-TGGGGAGATGTC TCTCTTTGA-3'; ii) circRNA-0012592 forward, 5'-TCGCAT CTACTGGTGTGACC-3' and reverse, 5'-TCAGTGCAGATG TGTGAGCA-3'; iii) circRNA-0087352 forward, 5'-TGATGC AGGAGATGATGAGG-3' and reverse, 5'-ATTATATCCCCC TGGGATGC-3'; iv) circRNA-0073009 forward, 5'-TTGCTA TGACTACATTTTGAGGTTTT-3' and reverse, 5'-TGGCCT CTCCGAAGTAGAAA-3'; v) circRNA-0008517 forward, 5'-GAATCAAACCTTGGGGACCT-3' and reverse, 5'-AAG GATGGGTTCAGGTAGGG-3'; vi) circRNA-0009054 forward, 5'-CTTTCCTCCAACAGCCACAC-3' and reverse, 5'-CTGCACGCTCTGTAGTCGAG-3'; vii) DNMT1 forward, 5'-CCTAGCCCCAGGATTACAAGG-3' and reverse, 5'-ACT CATCCGATTTGGCTCTTTC-3'; viii) GAPDH forward, 5'-CAATGACCCCTTCATTGACC-3' and reverse, 5'-TTG ATTTTGGAGGGATCTCG-3'; ix) miR1264 forward, 5'-CAA
GTCTTATTTGAGCACCTGTT-3' and Universal 5' primer 5'-GCGAGCACAGAATTAATACGAC-3'; x) U6 forward, 5'-CTCGCTTCGGCAGCACA-3' and reverse, 5'-AAC GCTTCACGAATTTGCGT-3'. The thermocycling conditions consisted of an initial denaturation for $2 \mathrm{~min}$ at $95^{\circ} \mathrm{C}$, followed by 40 cycles of $20 \mathrm{sec}$ at $95^{\circ} \mathrm{C}, 30 \mathrm{sec}$ at $60^{\circ} \mathrm{C}$, and $40 \mathrm{sec}$ at $72^{\circ} \mathrm{C}$. All reactions were performed in an ABI 7500 Real-Time fluorescence qPCR System (Applied Biosystems; Thermo Fisher Scientific, Inc.). GAPDH and U6 snRNA were used as the reference gene. Relative gene expression levels were calculated using the $2^{-\Delta \Delta \mathrm{Cq}}$ method (21).

Western blot analysis. Serum-Exos and HUVECs were collected and incubated on ice for 30 min using RIPA lysis buffer (Beyotime Institute of Biotechnology) containing protease inhibitor cocktail (Sigma-Aldrich; Merck KGaA). Protein quantification was performed by Pierce ${ }^{\mathrm{TM}}$ BCA Protein Assay Kit (Thermo Fisher Scientific, Inc.). The equivalent of $5 \mu \mathrm{g}$ protein were separated by $8-12 \%$ SDS-PAGE (Beijing Solarbio Science \& Technology Co., Ltd.), then transferred to PVDF (EMD Millipore). After blocking using $5 \%$ non-fat milk for $1 \mathrm{~h}$ at room temperature, the membrane was incubated with 1:1,000 dilution of primary antibodies against CD63 (cat. no. ab134045), CD9 (cat. no. ab92726), TSG101 (cat. no. ab125011), phosphorylated (p)-STAT3 (cat. no. ab76315), STAT3 (cat. no. ab68153), SOCS3 (cat. no. ab16030), DNMT1 (cat. no. ab188453) and GAPDH (cat. no. ab8245; reference protein) overnight at $4^{\circ} \mathrm{C}$. Next, the membrane was rinsed three times with TBS $+0.1 \%$ Tween-20 and incubated for $1 \mathrm{~h}$ with the horseradish peroxidase-conjugated goat anti-rabbit (ab205718; 1:5,000) or goat anti-mouse (ab205719; 1:5,000) secondary antibodies at ambient temperature. Finally, The signal of protein bands were visualized by Pierce $^{\mathrm{TM}}$ ECL Western Blotting Substrate (Thermo Fisher Scientific, Inc.) using the Tanon 4200 system (Tanon). The integrated density values were calculated using Quantity One v4.6.6 software (Bio-Rad Raboratories). All antibodies were purchased from Abcam.

MTT assay. HUVECs were seeded in 96-well plates at a density of $5 \times 10^{4}$ cells $/ \mathrm{ml}$ in a100- $\mu 1$ volume of conditioned medium. Experimental samples were treated with $50 \mu \mathrm{g} / \mathrm{ml}$ UA-Exos or SA-Exos for 24 or $48 \mathrm{~h}$, while mock samples were treated with PBS. Blank wells were prepared by adding $100 \mu \mathrm{l}$ of culture medium to one well per plate. For each group, six replicates were set up in adjacent wells. After incubation, $20 \mu \mathrm{l}$ MTT (5 g/l) was added to each well, and the plate was incubated for an additional $4 \mathrm{~h}$ at $37^{\circ} \mathrm{C}$. Then, the medium in each well was removed, and $150 \mu \mathrm{l}$ of DMSO was added for $10 \mathrm{~min}$. The absorbance was measured in each well at $490 \mathrm{~nm}$ using a Multiskan Ascent instrument (Thermo Fisher Scientific, Inc.). Each experiment was repeated three times.

Wound healing assay. HUVECs were seeded in 6-well plates at a density of $1 \times 10^{6}$ cells/ $\mathrm{ml}$ in a 2 -ml volume of FBS-free RPMI-1640 medium. Experimental samples were treated with $50 \mu \mathrm{g} / \mathrm{ml}$ UA-Exos or SA-Exos, while mock samples were treated with PBS. When cell confluence reached $80-90 \%$, then a wound was created across the diameter of the well surface 
B

A

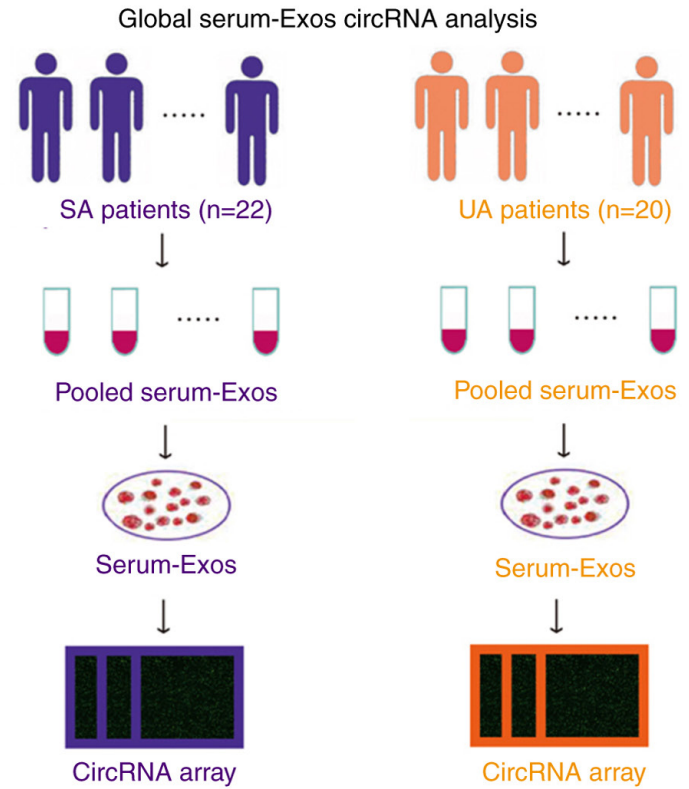

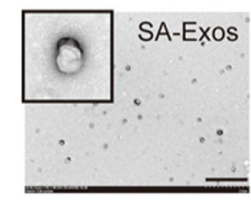

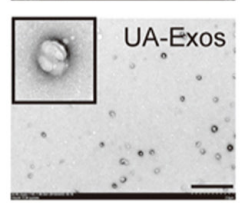

$\mathrm{D}$
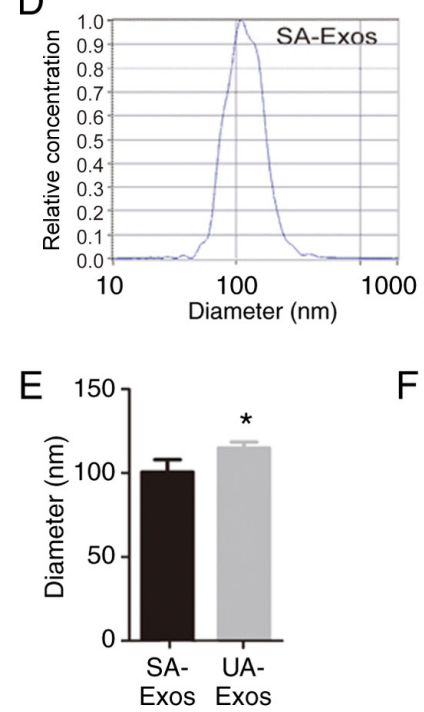

$\mathrm{F}$
C

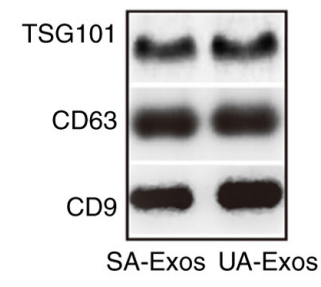

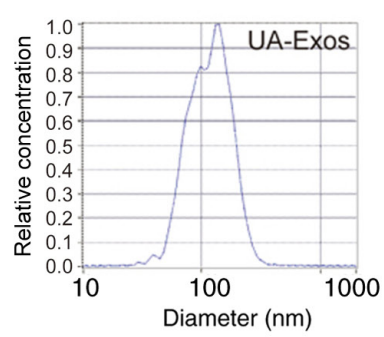

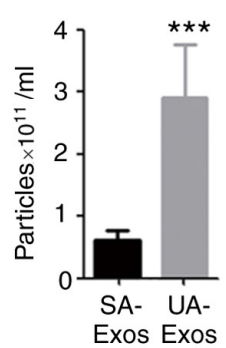

Figure 1. Identification of serum-Exos. (A) Flow chart of exosome isolation and circRNA analysis. (B) Morphology of serum-Exos visualized under a transmission electron microscope. Scale bar, $2.0 \mu \mathrm{m}$ (C) Western blot analysis of the exosome-specific markers TSG101, CD63 and CD9 in SA-Exos and UA-Exos. (D) Nanoparticle tracking analysis of exosome size distribution. (E) Diameter and (F) concentration of serum-Exos in the SA and UA groups. Data are presented as the mean $\pm \mathrm{SD}$. ${ }^{* * *} \mathrm{P}<0.001$, and ${ }^{*} \mathrm{P}<0.05 . \mathrm{n}=3$ in each group. Serum-Exos, serum exosomes; circRNA, circular RNA; SA, stable plaque atherosclerosis; UA, unstable/vulnerable plaque atherosclerosis.

with a pipette tip. After $24 \mathrm{~h}$, the wound area was measured under a light microscope (magnification, x100). The migration distance was measured at five locations per sample. The level of migration area was evaluated as follows: migration area $(\%)=(\mathrm{A} 0-\mathrm{A} 1) / \mathrm{A} 0 \times 100$, where $\mathrm{A} 0$ is the initial wound area and $\mathrm{A} 1$ is the wound area at $24 \mathrm{~h}$.

Transwell migration assay. Cell migration assays were performed using Transwell cell culture chambers (Thermo Fisher Scientific, Inc.). HUVECs were seeded into the upper chamber at a density of $2 \times 10^{4}$ cells $/ \mathrm{ml}$ in a $100-\mu \mathrm{l}$ volume of conditioned medium, to which $50 \mu \mathrm{g} / \mathrm{ml}$ UA-Exos or SA-Exos was added. The HUVECs treated with $50 \mu 1$ PBS were used as mock group. The lower chamber was loaded with $0.8 \mathrm{ml}$ medium supplemented with $10 \%$ exosome-free FBS. The Transwell plates were incubated at $37^{\circ} \mathrm{C}$ and $5 \% \mathrm{CO}_{2}$ for $24 \mathrm{~h}$. After incubation, the filters were stained with $0.1 \%$ crystal violet, then decolorized with $33 \%$ acetic acid (22). Finally, the absorbance was measured at $570 \mathrm{~nm}$ using a Multiskan Ascent instrument (Thermo Fisher Scientific, Inc.) to evaluate the migratory ability of HUVECs.

Statistical analysis. SPSS 16.0 (SPPS, Inc.) was used for statistical analysis. The distribution of the data was determined using the Kolmogorov-Smirnov test. Normally distributed data are presented as the mean $\pm \mathrm{SD}$, and were analyzed using an unpaired Student's t-test, or one-way ANOVA followed by Bonferroni correction. Data with a skewed distribution are presented as the medians and interquartile ranges (IQR), and were analyzed using Mann-Whitney's U test. Categorical variables are presented as counts or percentages, and were analyzed using the $\chi^{2}$ test. Correlations between serum-Exo-circRNA-0006986 and clinical biochemical parameters were evaluated using Spearman's rank correlation analysis. Scientific graphs were generated using GraphPad Prism (version 5.0; GraphPad Software, Inc.). P $<0.05$ was considered to indicate a statistically significant difference.

\section{Results}

Demographic and baseline characteristics of the study population. A total of 22 patients with SA (age range, 37-57) and 20 patients with (age range, 41-65) were enrolled in this study. Their baseline clinical and biochemical characteristics are summarized in Table I. The concentration the concentration of low-density lipoprotein cholesterol (LDL-C) and C-reactive protein (CRP) were significantly increased in the UA group, compared with the SA group. No significant differences in age, 
A

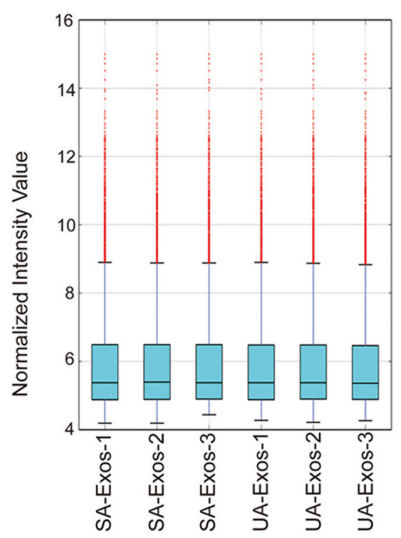

C

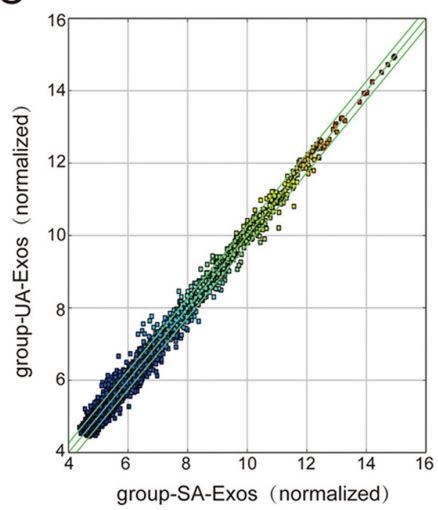

B

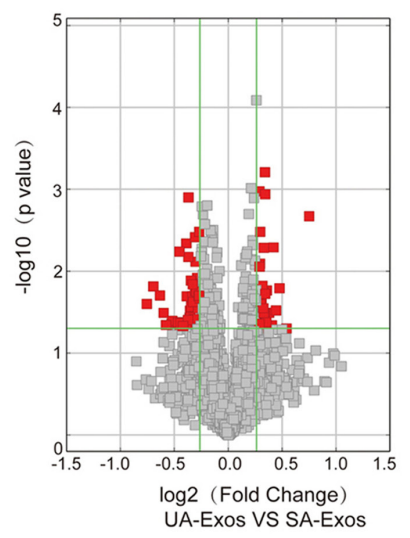

$\mathrm{D}$

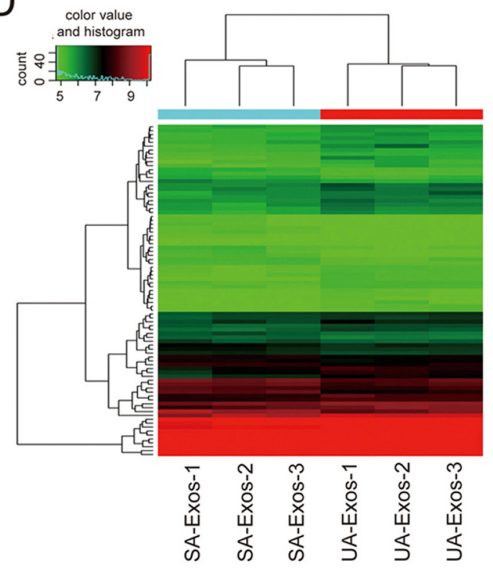

Figure 2. circRNA microarray expression data in serum-Exos (A) Box plots displaying the variations in circRNA expression after normalization. (B) Volcano plot of circRNAs. The vertical lines correspond to 1.2-fold up and down, respectively, and the horizontal line represents the P-value cut-off of 0.05 . Statistically significant differentially expressed circRNAs are represented by red symbols. (C) Scatter plot of circRNA expression. Symbols above the upper green line or below the lower green line represent circRNAs with a change in expression between the SA-Exos and US-Exos groups >1.2-fold. (D) Hierarchical clustering of circRNA expression across the SA-Exos and UA-Exos groups. Gene expression profiles are shown in rows. Red indicates upregulated expression of circRNAs, and green indicates downregulated expression of circRNAs. Exos, exosomes; circRNA, circular RNA; SA, stable plaque atherosclerosis; UA, unstable/vulnerable plaque atherosclerosis.

sex, smoking status, or any other biochemical parameter were observed between the two groups.

Exosome isolation and identification. Serum samples from patients with SA or UA were pooled for exosome extraction (Fig. 1A). Under the transmission electron microscope, the exosomes exhibited a double-membrane structure in both groups, and the diameters of most exosomes were $<200 \mathrm{~nm}$ (Fig. 1B). Moreover, the exosomes expressed the exosomal markers CD9, CD63, and TSG101 (Fig. 1C). The concentration of exosomes in the SA group was significantly increased, compared with the UA group $(\mathrm{P}<0.001)$. However, their diameters were only marginally different in the two groups $(\mathrm{P}<0.05$; Fig. 1D-F).

circRNA profile overview. The expression profile of Exos-circRNAs was determined using microarray analysis. The expression levels of circRNA molecules were normalized to the same order of magnitude prior to the statistical analysis. The median expression levels of different groups nearly the same following normalization, indicating a great degree of standardization (Fig. 2A). A total of 75 significant differentially expressed circRNAs in the UA-Exos and SA-Exos groups are displayed as a volcano plot in Fig. 2B. A scatter plot demonstrated the variability of 5,343 circRNAs expression in UA-Exos and SA-Exos (Fig. 2C). The expression levels of all 5,343 circRNAs in UA-Exos and SA-Exos were quantified and visualized on a hierarchical clustering heat map. Only 37 circRNAs were upregulated and 48 were downregulated in the UA-Exos group, compared with SA-Exos, indicating a strikingly similar expression pattern of circRNAs between these two groups (Fig. 2D).

RT-qPCR validation, prediction of $m R N A-m i R N A-\operatorname{circ} R N A$ relationships and enrichment analysis of the biological functions of circRNA-0006896-co-expressed genes. To verify the microarray profiling expression data, the expression of the three most upregulated (circRNA-0006896, circRNA-0012592 and circRNA-0087352) or downregulated circRNAs (circRNA-0073009, circRNA-0008517 and circRNA-0009054) was validated using RT-qPCR. Among these circRNAs, the circRNA-0006896 was the only one that expressed over $\sim 2$-fold UA-Exos samples relative to SA-Exos (Fig. 3A). Therefore, circRNA-0006896 was selected as a candidate for subsequent experiments. Moreover, the expression trend of circRNA-0006896 was further verified 
A

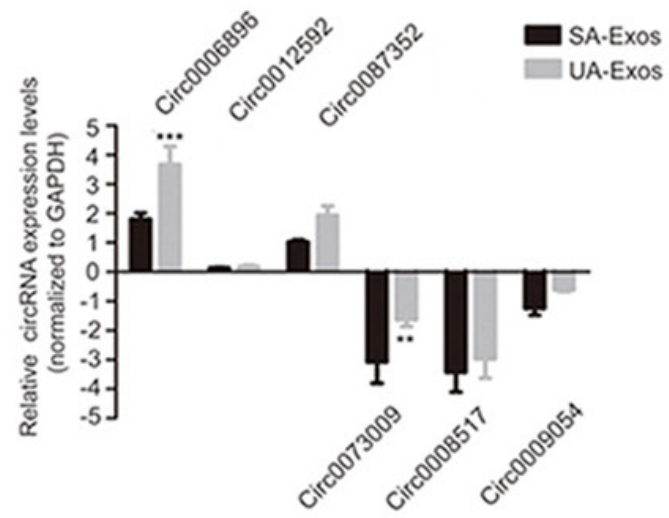

B

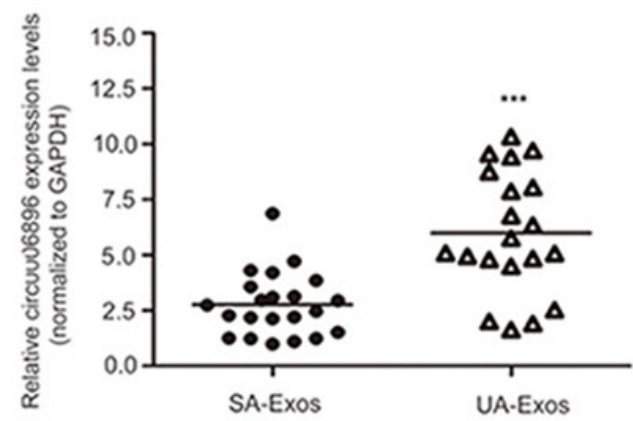

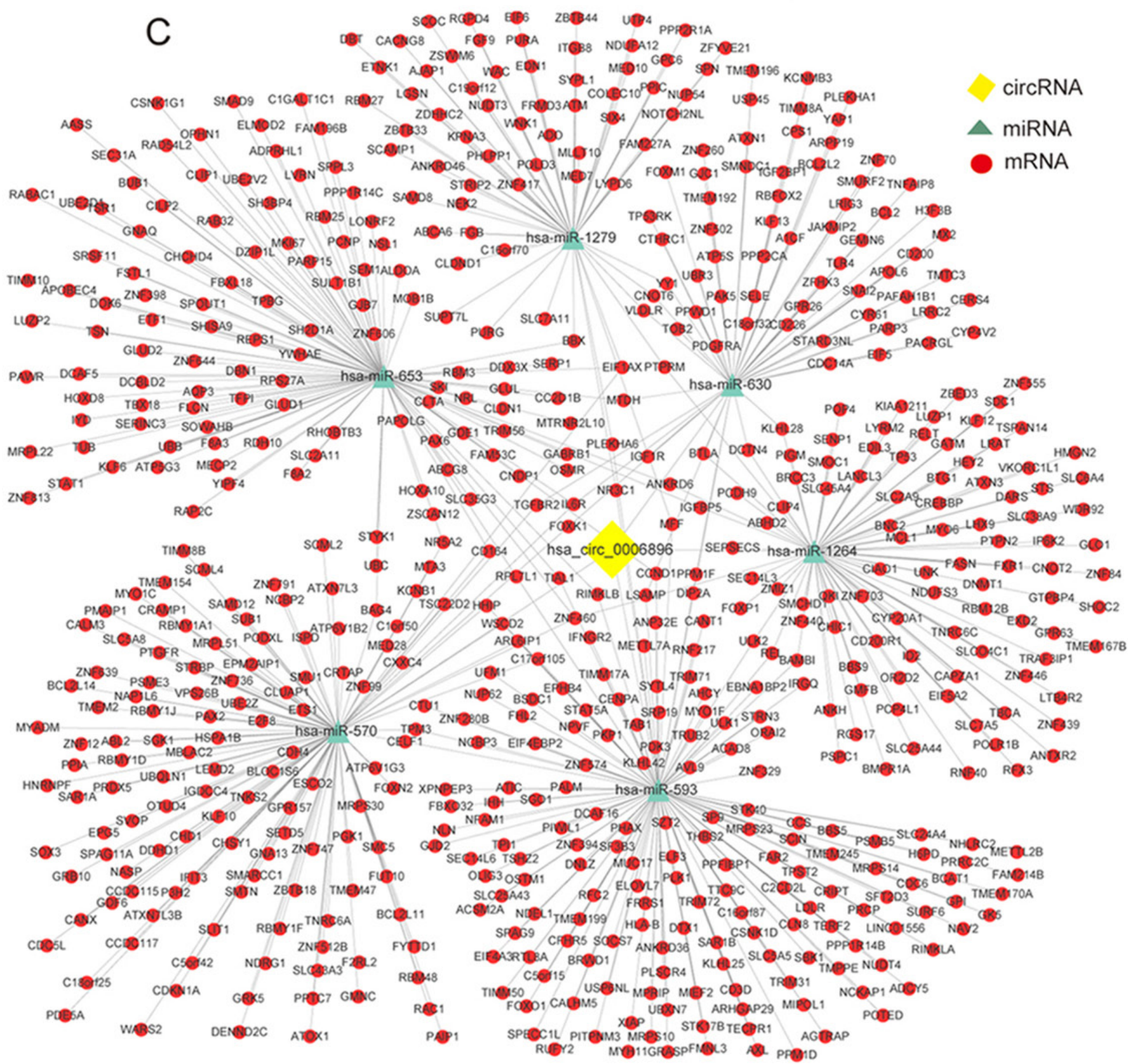

Figure 3. Validation of expression patterns for circRNAs and construction of the ceRNA network. (A) RT-qPCR validation of the expression patterns of six circRNA candidates. (B) RT-qPCR validation of circRNA-0006896 expression in patients with SA or UA. (C) circRNA-0006896 ceRNA network diagram. Yellow diamonds represent the circRNAs, blue triangles correspond to the target miRNAs, and red circles represent miRNA-targeted mRNAs. RT-qPCR was performed in triplicate. Data are presented as the mean $\pm \mathrm{SD} .{ }^{* * *} \mathrm{P}<0.001$ and ${ }^{* *} \mathrm{P}<0.01$. Exos, exosomes; circRNA, circular RNA; miRNA, microRNA; SA, stable plaque atherosclerosis; UA, unstable/vulnerable plaque atherosclerosis; RT-qPCR, reverse transcription-quantitative PCR. 
Table II. Correlation between circRNA-0006896 expression and biochemical parameters in AS patients with SA or UA.

\begin{tabular}{lrrrrr}
\hline & \multicolumn{2}{c}{$\mathrm{SA}(\mathrm{n}=22)$} & & \multicolumn{2}{c}{$\mathrm{UA}(\mathrm{n}=20)$} \\
\cline { 2 - 3 } \cline { 5 - 6 } $\begin{array}{l}\text { Biological } \\
\text { parameters }\end{array}$ & $\mathrm{r}$ & $\mathrm{P}$-value & & $\mathrm{r}$ & P-value \\
\hline TC & 0.454 & 0.161 & & 0.191 & 0.421 \\
TG & -0.006 & 0.986 & & 0.653 & 0.002 \\
HDL-C & 0.126 & 0.713 & & -0.505 & 0.136 \\
LDL-C & 0.432 & 0.018 & & 0.547 & 0.008 \\
GLU & 0.109 & 0.749 & & 0.113 & 0.755 \\
GHb & -0.225 & 0.561 & & -0.421 & 0.226 \\
Uric acid & 0.276 & 0.412 & & 0.662 & 0.052 \\
ALT & -0.420 & 0.199 & & -0.023 & 0.915 \\
ALB & -0.579 & 0.062 & & -0.927 & $<0.001$ \\
AST & 0.392 & 0.081 & & -0.876 & 0.783 \\
BUN & 0.088 & 0.797 & & 0.454 & 0.187 \\
GGT & -0.448 & 0.167 & & -0.250 & 0.486 \\
sCr & 0.334 & 0.129 & & 0.287 & 0.220 \\
CRP & 0.145 & 0.536 & 0.532 & 0.016 \\
\hline
\end{tabular}

TC, total cholesterol; TG, triglyceride; HDL-C, high-density lipoprotein cholesterol; LDL-C, low-density lipoprotein cholesterol; GLU, glucose levels upon admission; GHb, glycated hemoglobin; ALT, alanine aminotransferase; ALB, serum albumin; AST, aspartate aminotransferase; BUN, blood urea nitrogen; GGT, $\gamma$-glutamyl transpeptidase; sCr, serum creatinine; CRP, C-reactive protein; $\mathrm{SA}$, stable plaque atherosclerosis; UA, unstable/vulnerable plaque atherosclerosis; $r$, Pearson's correlation coefficient.

in individual serum-Exos samples from patients with SA $(n=22)$ and UA $(n=20)$. As shown in Fig. 3B, the expression of circRNA-0006896 was significantly increased in serum-Exos from UA patients, compared with SA patients. These results suggested that circRNA-0006896 may be involved in plaque instability.

Furthermore, circRNAs can serve as a competitive endogenous RNA (ceRNA) to sponge miRNAs to regulate the target mRNAs $(14,15)$. To describe the role of circRNA-0006896 during the progression of carotid plaque destabilization, bioinformatics analysis was used to predict the potential target miRNAs of circRNA-0006896 and construct a circRNA-miRNA network map associated with AS. This analysis predicted seven target miRNAs (miR-1264, miR-1279, miR-570, miR-593, miR-630 and miR-653) for circRNA-0006896. A ceRNA network map was then constructed, which contained one circRNA, six miRNAs and 768 mRNAs (Fig. 3C). This network map revealed that the upregulation of circRNA-0006896 in UA -Exos might upregulate the expression levels of 122 mRNA molecules by reducing the expression of miR1264, upregulate 118 mRNA targets by reducing the expression of miR1279, upregulate 70 mRNA targets by reducing the expression of miR570, upregulate 285 mRNA targets by reducing the expression of miR593, upregulate 96 mRNA targets by reducing the expression of miR630 and upregulate 194 mRNA targets by reducing the expression of miR653 (Fig. 3C).
Correlations between circRNA-006896 serum-Exos levels with biochemical parameters in AS patients. To further examine the role of circRNA-0006896 in the progression of carotid plaque instability, Spearman's rank correlation analysis was carried out to determine whether exosomal circRNA-0006896 levels were associated with biochemical parameters in patients with SA or UA. circRNA-006896 levels in serum-Exos were positively correlated with LDL-C levels in the SA group. Moreover, in the UA group, they were also positively correlated with triglyceride, LDL-C and CRP levels, but negatively correlated with albumin levels. Moreover, no statistically significant correlations were observed between the serum-Exo-circRNA-006896 level and the levels of total cholesterol, high density lipoprotein cholesterol, glucose, glycated hemoglobin, uric acid, alanine aminotransferase, aspartate aminotransferase, blood urea nitrogen, $\gamma$-glutamyl transpeptidase, or creatinine in either the UA or SA group (Table II). These findings suggested that the increased expression of circRNA-006896 in UA serum-Exos was positively correlated with the levels of TG, LDL-C and CRP in UA patients.

UA-Exos influence the circRNA-0006896-miR-1264-DNMT1 axis in HUVECs. A previous study reported that HUVEC proliferation and migration are important events that induce plaque destabilization $(23,24)$. The signaling molecules carried by exosomes may affect the biological behavior of target cells in the microenvironment (25). Therefore, it was hypothesized that endothelial cell phagocytosis of serum-Exos containing high levels of circRNA-0006896 could promote endothelial cell proliferation and migration, leading to plaque instability. According to the ceRNA network hypothesis, circRNAs can abolish the inhibitory effect of miRNAs on their target genes and cause changes in intracellular signaling pathways (26). Thus, in this study, the role of the circRNA-0006896 ceRNA network in HUVECs following treatment with serum-Exos was further examined.

RT-qPCR analysis suggested that miR1264 was the most downregulated of the six circRNA-0006896-targeted miRNAs following treatment with UA-Exos (Fig. 4A). A previous report indicated that miR-1264 targets the DNMT1 transcript by binding to its 3 'UTR, thus affecting DNMT1 expression and enzyme activity in miR-1264-transfected cells $(27,28)$. Thus, it was hypothesized that circRNA-0006896 may regulate HUVEC behavior through a circRNA-0006896-miR1264-DNMT1 axis. The miRanda database suggested that the 3'-UTR of circRNA-0006896 contains a putative direct binding site for miR1264 (Fig. 4B). Dual-luciferase reporter assays indicated that co-transfection of WT circRNA-0006896 with miR1264 mimics would lead to a significant reduction in fluorescence intensity, compared with co-transfection with control miRNA mimics. However, relative fluorescence intensity was not changed when the MUT circRNA-0006896 construct was co-transfected, indicating the specific miR1264 sponges function of circRNA-0006896 (Fig. 4C). In addition, confocal microscopy images indicated that the amount of exosomes phagocytosed by HUVECs did not appear different between the SA and UA-Exos groups (Fig. 4D). FISH also confirmed that circRNA-0006896 colocalized with miR-1264 around the nuclear membrane (Fig. 4E). 
A

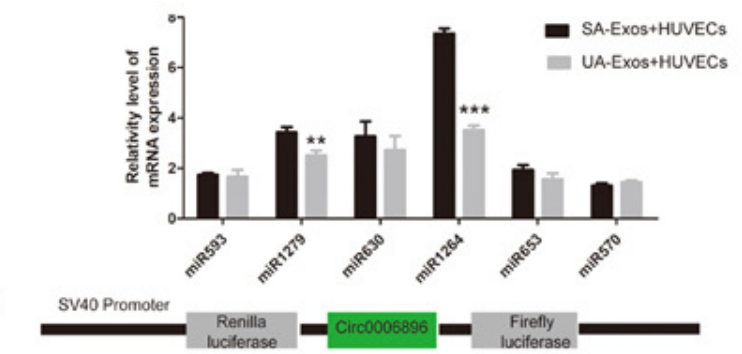

WT circ0006896 5'...CCAUACAUUUCAAUU-..AGACUUAU....3' miR1264 3'...UUGUCCACGAGUUUAUUCUGAAC...5'

Mutant circ0006896 5'...CCAUACAUUAGUUUU-..UCUGAAAU....3'

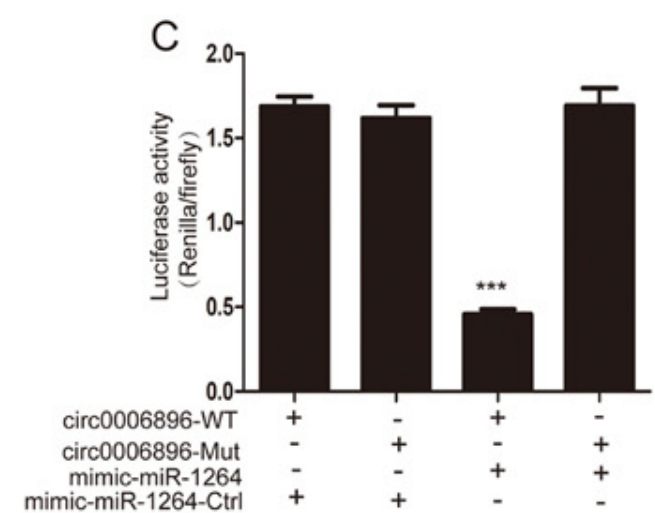

D
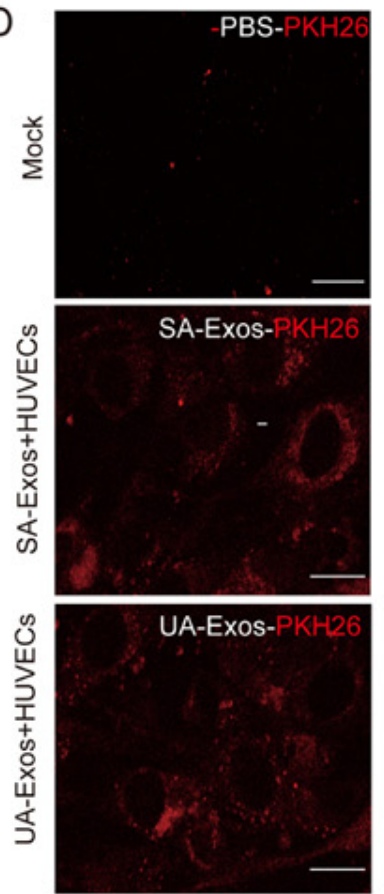

F

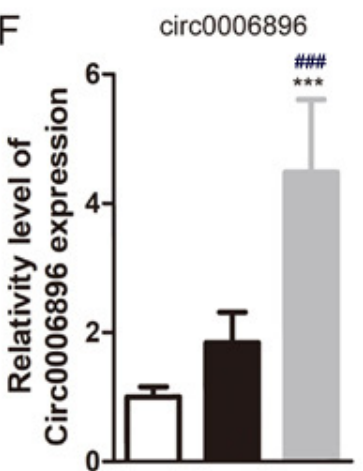

E
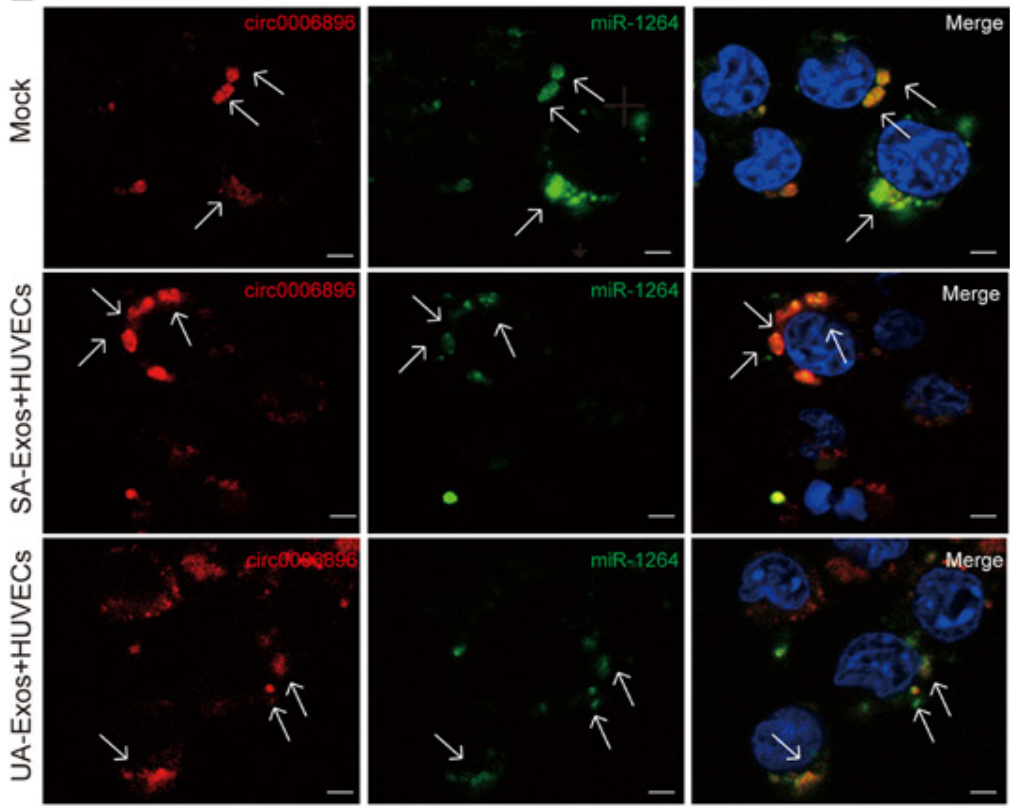

G

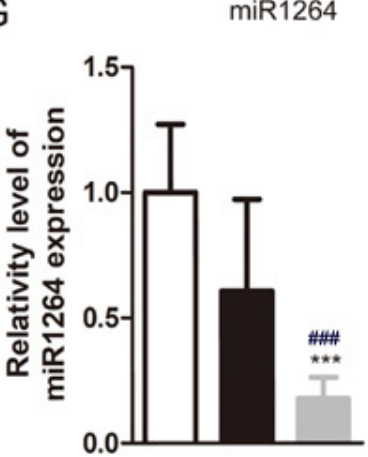

H DNMT1 mRNA

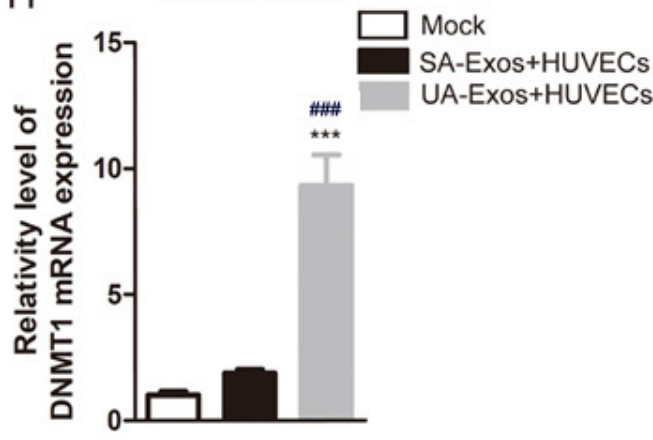

Figure 4. circRNA-006896-miR1264-DNMT1 axis in HUVECs. (A) RT-qPCR validation of the expression patterns of six circRNA-006896 targeted-miRNAs in SA-Exos or UA-Exos-treated HUVECs. (B) Predicted formation of duplexes at the miR1264 binding sites in circRNA-0006896 is indicated. (C) A Dual-luciferase reporter gene assay was carried out to verify the binding site. HUVECs treated with miR-1264 mimic or control miRNA were co-transfected with psiCHECK 2 constructs containing the WT or mutant circRNA-0006896 sites. (D) Confocal microscopy analysis of serum-Exos phagocytosis by HUVECs. (E) Colocalization of circRNA-0006896 with miR1264 in HUVECs Fluorescence in situ hybridization was used to evaluate the cellular localization of circRNA-0006896 and miR-1264. Nuclei were stained with DAPI. Relative expression levels of (F) circRNA-0006896, (G) miR-1264 and (H) DNMT1 in the mock and serum-Exos-treated HUVECs. Scale bar, $100 \mu \mathrm{m}$. Data are presented as the mean \pm SD. ${ }^{* * *} \mathrm{P}<0.001,{ }^{* *} \mathrm{P}<0.01,{ }^{\# \# \#} \mathrm{P}<0.001$ vs. SA-Exos. Serum-Exos, serum exosomes; circRNA, circular RNA; miRNA, microRNA; HUVEC, human umbilical vein endothelial cell; DNMT1, DNA methyltransferase 1; WT, wild-type; Mut, mutant; SA, stable plaque atherosclerosis; UA, unstable/vulnerable plaque atherosclerosis.

Furthermore, the expression levels of circRNA-0006896, miR1264 and DNMT1 were measured in HUVECs following treatment with serum-Exos from patients with UA or SA. In the UA group, the expression levels of both circRNA-0006896 

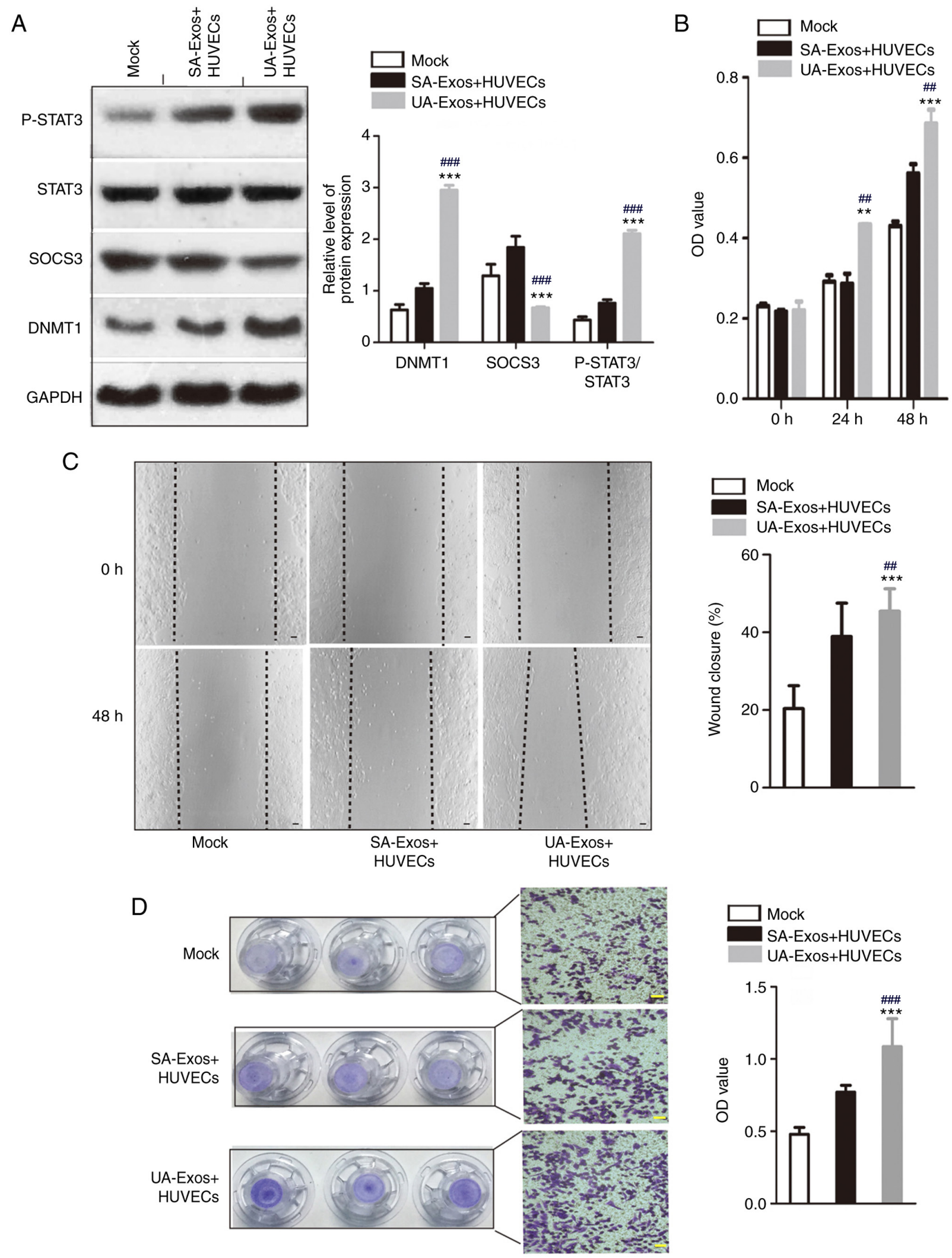

Figure 5. Highly expressed circRNA-0006896 encapsulated in UA-serum-Exos increases the proliferation and migration of HUVECs. (A) Protein levels of DNMT1, SOCS3, P-STAT3 and STAT3 were determined by western blot analysis. (B) MTT assay. (C) Wound healing assay. (D) Transwell assay. Scale bar, $100 \mu \mathrm{m}$. Data are presented as the mean $\pm \mathrm{SD}$. ${ }^{* * * *} \mathrm{P}<0.001,{ }^{* * *} \mathrm{P}<0.01$ vs. mock group; ${ }^{\# \# *} \mathrm{P}<0.001,{ }^{\# t} \mathrm{P}<0.01$ vs. SA-Exos. Exos, exosomes; HUVEC, human umbilical vein endothelial cell; DNMT1, DNA methyltransferase 1; SOCS3, suppressor of cytokine signaling 3; signal transducer and activator of transcription 3; P, phosphorylated; SA, stable plaque atherosclerosis; UA, unstable/vulnerable plaque atherosclerosis; OD, optical density.

and DNMT1 were significantly increased, compared with the SA group. In addition, the expression of miR-1264 was significantly lower, compared with the SA group (Fig. 4F-H). Therefore, the expression levels of circRNA-0006896, 
DNMT1 and miR-1264 differed following treatment with SA or UA-Exos. These data indicated that the high levels of circRNA-0006896 contained within UA-Exos can affect the expression of miR-1264 and DNMT1 in HUVECs, whereas the small amounts of circRNA-0006896 contained in SA-Exos do not.

The circRNA-0006896 network in UA exosomes increases the proliferation and migration of HUVECs. Previous studies have documented that DNMT1 is a critical regulator of JNK, STAT, and NF- $\mathrm{B}$ signaling during the development of UA because an increase DNMT1 expression results in $\mathrm{CpG}$ island hypermethylation in the promoter region of SOCS3, which downregulates its expression (28). Low expression of SOCS3 promotes JNK/STAT signaling, thus increasing the proliferation and migration of HUVECs (29), which in turn leads to plaque destabilization and promotes AS development $(23,30,31)$. Thus, functional assays using HUVECs treated with UA or SA-Exos were carried out to explore the role of circRNA-0006896, miR-1264 and DNMT1 in AS development.

Western blot analysis suggested that there was an inverse relationship between SOCS3 and DNMT1 expression. Indeed, SOCS3 protein expression was reduced in HUVECs following treatment with UA-Exos, compared with SA-Exos and mock groups. This was accompanied by a significant increase in STAT3 phosphorylation (Fig. 5A). In addition, the proliferation of HUVECs was significantly increased in the UA-Exos group, compared with the SA serum-Exos and mock groups at 24 and 48 h. (Fig. 5B). A wound healing assay was conducted to investigate the effects of circRNA-006896 on HUVEC migration. HUVEC migration increased by $20 \%$ in the UA-Exos group, compared with the SA-Exos and mock groups (Fig. 5C). In a Transwell assay, staining intensity significantly increased for HUVECs after treatment with UA-Exos for $48 \mathrm{~h}$, compared with the UA and mock groups, suggesting increased HUVEC migration (Fig. 5D). Collectively, these results suggested that high concentrations of circRNA-0006896 in UA-Exos enhances the proliferation and migration of HUVECs, possibly through DNMT1/SOCS3/JNK/STAT3 signaling.

\section{Discussion}

Previous studies have been conducted to identify therapeutic targets for UA, most of which have been limited to protein-coding genes, miRNAs and lncRNAs (32-35). Recently, circRNAs have attracted attention as new diagnostic markers for diseases, including cancer. circRNAs constitute a rich, stable, diverse and conserved family of RNA molecules. Emerging evidence suggests that circRNAs participate in various biological processes, such as angiogenesis, proliferation, and differentiation (36). Recently, Zhang et al (37) demonstrated that the crosstalk between circRNAs and their competing mRNAs might play crucial roles in the development of UA by regulating cell adhesion, migration, and activation. Moreover, exosomal circRNAs derived from peripheral circulating serum are associated with UA formation. Jiang et al (38) reported that peripheral circulating serum-Exos are can induce TNF- $\alpha$ and IL- 6 production in macrophages through their miRNA cargo.
An increase in inflammatory cytokines has been proven to be related to plaque vulnerability across various stages of AS, in which vascular endothelial cells are dysfunctional and induced by stimulatory factors. Endothelial cell functions, such as antithrombogenicity and angiogenic sprouting capacity, are lost. However, mesenchymal cell characteristics, such as contractility, proliferation, and migration, are enhanced. Functionally, endothelial cells play a vital role in the development and ultimate rupture of unstable plaques, which cause most acute coronary artery events $(3,23)$. However, few studies have explored the stimulatory effect of peripheral circulating serum-Exos on the vascular endothelium in AS patients.

Therefore, in the present study, the circRNA expression profiles of serum-Exos from patients with SA or UA were used to assess the effect of exosomal circRNAs on vascular endothelial cell activation. circRNA-0006896 was selected for further study due to its higher expression in patients with UA, compared with patients with SA. Furthermore, the expression levels of circRNA-0006896 were positively correlated with the levels of triglyceride, LDL-C and CRP, and negatively correlated with albumin levels in patients with UA. Since increased levels of serum LDL-C and CRP are an important clinical feature of UA formation, it was hypothesized that serum-Exo-circRNA-0006896, which is positively correlated with their expression, could play an important role in the formation of unstable carotid plaques.

The circRNA-0006896-miR-1264-DNMT1 axis was selected as a platform to study the regulatory roles of circRNA-0006896 in serum-Exos through circRNA bioinformatic analysis. The results indicated that following treatment with UA serum-Exos, the expression of circRNA-0006896 in HUVECs was upregulated, which was accompanied by downregulated expression of miR1264 and upregulated expression of DNMT1 mRNA. Recently, DNMT1 has been identified as the direct target of miR1264, which can bind to the 3'UTR of the DNMT1 transcript to inhibit its expression. DNMT1 is an important enzyme implicated in DNA methylation, particularly in the promoter region of SOCS3. Moreover, $\mathrm{CpG}$ islands, one of which is located at $\mathrm{kb} 4.261-6.673$ in the SOCS3 genomic locus, regulate DNMT1 function $(27,28)$. miR-1264 downregulation facilitates persistent DNMT1 expression, thereby inducing SOCS3 promoter methylation and impacting its gene expression (27). Downregulated expression of SOCS3 results in loss of its inhibitory effect on the JNK/STAT3 pathway $(30,31)$. The proliferation and migration of HUVECs were increased most significantly in the UA groups compared to the SA and mock groups, possibly indicating its role in the formation of unstable plaques.

In conclusion, increased expression of circRNA-0006896 in serum-Exos from patients with UA promotes the proliferation and migration of HUVECs by negatively regulating the expression of miR-1264. In turn, this leads to an increase in DNMT1 expression and STAT3 phosphorylation, and a reduction in the expression of SOCS3. These findings indicate that circRNA-0006896 is a potential therapeutic target that regulates JNK/STAT3 pathway activation and may influence vulnerable plaque formation by forming complex regulatory networks in patients with UA. Therefore, this study may 
provide insight into novel interventions against vulnerable plaque formation in patients with UA.

\section{Acknowledgements}

Not applicable.

\section{Funding}

The present study were supported by the Shenzhen Sciences and Technology Project Foundation (grant no. JCYJ201700818162010186) and the Public welfare research project in Futian District, Shenzhen (grant. no. FTWS2018073).

\section{Availability of data and materials}

The data and materials of this study are available from the corresponding author upon reasonable request

\section{Authors' contributions}

YW and ZHJ conceived and designed the experiments. YC, YML, ZQL, CJ and ZWY performed the experiments. CYX, ZWQ, LH and LSJ collected and analyzed the data. YW and CJ were the major contributor in writing the manuscript. All authors read and approved final version of the manuscript.

\section{Ethics approval and consent to participate}

The present study was approved by the Ethics Revie Committee of the Eighth Affiliated Hospital of Sun Yat-Sen Unive rsity (Shenzhen, China).

\section{Patient consent for publication}

All participants have given their written permission for publication of obtained data.

\section{Competing interests}

The authors declare that they have no competing interests.

\section{References}

1. Getz GS and Reardon CA: Atherosclerosis: Cell biology and lipoproteins. Curr Opin Lipidol 31: 286-290, 2020.

2. Harari F, Barregard L, Östling G, Sallsten G, Hedblad B, Forsgard N, Borné Y, Fagerberg B and Engström G: Blood lead levels and risk of atherosclerosis in the carotid artery: Results from a swedish cohort. Environ Health Perspect 127: 127002, 2019.

3. Schiro A, Wilkinson FL, Weston R, Smyth JV, Serracino-Inglott F and Alexander MY: Elevated levels of endothelial-derived microparticles, and serum CXCL9 and SCGF- $\beta$ are associated with unstable asymptomatic carotid plaques. Sci Rep 5: 16658, 2015.

4. Ferronato S, Mombello A, Posenato I, Candiani P, Scuro A, Setacci $\mathrm{C}$ and Gomez-Lira M: Expression of circulating miR-17-92 Cluster and HDAC9 gene in atherosclerotic patients with unstable and stable carotid plaques. Genet Test Mol Biomarkers 21: 402-405, 2017.

5. Wang M, Su P, Liu Y, Zhang X, Yan J, An X, Wang X and Gu S: Abnormal expression of circRNA_089763 in the plasma exosomes of patients with postoperative cognitive dysfunction after coronary artery bypass grafting. Mol Med Rep 20: 2549-2562, 2019.
6. Wang Y, Xie Y, Zhang A, Wang M, Fang Z and Zhang J: Exosomes: An emerging factor in atherosclerosis. Biomed Pharmacother 115: 108951, 2019.

7. Wang Y, Xie Y, Zhang A, Wang M, Fang Z and Zhang J: Corrigendum to 'Exosomes: An emerging factor in atherosclerosis'. Biomed Pharmacother 118: 109119, 2019.

8. Dumache R, Ciocan V, Muresan C, Rogobete AF and Enache A: Circulating MicroRNAs as promising biomarkers in forensic body fluids identification. Clin Lab 61: 1129-1135, 2015.

9. Cortez MA, Bueso-Ramos C, Ferdin J, Lopez-Berestein G, Sood AK and Calin GA: MicroRNAs in body fluids-the mix of hormones and biomarkers. Nat Rev Clin Oncol 8: 467-477, 2011.

10. Kuner R, Brase JC, Sultmann H and Wuttig D: microRNA biomarkers in body fluids of prostate cancer patients. Methods 59: 132-137, 2013.

11. Goetzl EJ, Goetzl L, Karliner JS, Tang N and Pulliam L: Human plasma platelet-derived exosomes: Effects of aspirin. FASEB J 30: 2058-2063, 2016.

12. Lu M, Yuan S, Li S, Li L, Liu M and Wan S: The exosome-derived biomarker in atherosclerosis and its clinical application. J Cardiovasc Transl Res 12: 68-74, 2019.

13. Geng X, Jia Y, Zhang Y, Shi L, Li Q, Zang A and Wang H: Circular RNA: Biogenesis, degradation, functions and potential roles in mediating resistance to anticarcinogens. Epigenomics 12: 267-283, 2020.

14. Wang L, Zheng Z, Feng X, Zang X, Ding W, Wu F and Zhao Q: circRNA/lncRNA-miRNA-mRNA network in oxidized, low-density, lipoprotein-induced foam cells. DNA Cell Biol 38: 1499-1511, 2019.

15. Luo J, Liu H, Luan S and Li Z: Guidance of circular RNAs to proteins' behavior as binding partners. Cell Mol Life Sci 76: 4233-4243, 2019.

16. Kuret T, Sodin-Semrl S, Mrak-Poljsak K, Cucnik S, Lakota K and Erman A: Interleukin-1 $\beta$ induces intracellular serum amyloid A1 expression in human coronary artery endothelial cells and promotes its intercellular exchange. Inflammation 42: 1413-1425, 2019.

17. Marchio P, Guerra-Ojeda S, Vila JM, Aldasoro M, Victor VM and Mauricio MD: Targeting early atherosclerosis: A focus on oxidative stress and inflammation. Oxid Med Cell Longev 2019: 8563845,2019

18. Lima Junior JC, Moura-Assis A, Cintra RM, Quinaglia T, Velloso LA and Sposito AC: Central role of obesity in endothelial cell dysfunction and cardiovascular risk. Rev Assoc Med Bras (1992) 65: 87-97, 2019.

19. Tanemura H, Maeda M, Ichikawa N, Miura Y, Umeda Y, Hatazaki S, Toma N, Asakura F, Suzuki H, Sakaida H, Matsushima S and Taki W: High-risk plaque for carotid artery stenting evaluated with 3-dimensional T1-weighted gradient echo sequence. Stroke 44: 105-110, 2013.

20. Yamada K, Yoshimura S, Kawasaki M, Enomoto Y, Asano T, Hara A, Minatoguchi S and Iwama T: Embolic complications after carotid artery stenting or carotid endarterectomy are associated with tissue characteristics of carotid plaques evaluated by magnetic resonance imaging. Atherosclerosis 215: 399-404, 2011.

21. Livak KJ and Schmittgen TD: Analysis of relative gene expression data using real-time quantitative PCR and the 2(-Delta Delta C(T)) method. Method 25: 402-408, 2001.

22. Wang Y, Shao S, Luo M, Huang S, Feng L, Yuan N, Wu F, Dang C and Zhao X: Effects of rat bone marrow-derived mesenchymal stem cells on breast cancer cells with differing hormone receptor status. Oncol Lett 14: 7269-7275, 2017.

23. Yang XP, Irani K, Mattagajasingh S, Dipaula A, Khanday F, Ozaki M, Fox-Talbot K, Baldwin WM III and Becker LC: Signal transducer and activator of transcription 3alpha and specificity protein 1 interact to upregulate intercellular adhesion molecule-1 in ischemic-reperfused myocardium and vascular endothelium. Arterioscler Thromb Vasc Biol 25: 1395-1400, 2005.

24. Chen LY, Wang X, Qu XL, Pan LN, Wang ZY, Lu YH and Hu HY: Activation of the STAT3/microRNA-21 pathway participates in angiotensin II-induced angiogenesis. J Cell Physiol 234: 19640-19654, 2019.

25. Maia J, Caja S, Strano Moraes MC, Couto N and Costa-Silva B: Exosome-based cell-cell communication in the tumor microenvironment. Front Cell Dev Biol 6: 18, 2018.

26. Awasthi R, Singh AK, Mishra G, Maurya A, Chellappan DK, Gupta G, Hansbro PM and Dua K: An overview of circular RNAs. Adv Exp Med Biol 1087: 3-14, 2018. 
27. Boosani CS, Dhar K and Agrawal DK: Down-regulation of hsa-miR-1264 contributes to DNMT1-mediated silencing of SOCS3. Mol Biol Rep 42: 1365-1376, 2015.

28. Boosani CS, Gunasekar P, Block M, Jiang W, Zhang Z, Radwan MM and Agrawal DK: Inhibition of DNA methyltransferase-1 instigates the expression of DNA methyltransferase-3a in angioplasty-induced restenosis. Can J Physiol Pharmacol 96: 1030-1039, 2018.

29. Li S, Geng Q, Chen H, Zhang J, Cao C, Zhang F, Song J, Liu C and Liang W: The potential inhibitory effects of miR $19 \mathrm{~b}$ on vulnerable plaque formation via the suppression of STAT3 transcriptional activity. Int J Mol Med 41: 859-867, 2018.

30. Santana FPR, da Silva RC, Grecco SDS, Pinheiro AJMCR, Caperuto LC, Arantes-Costa FM, Claudio SR, Yoshizaki K, Macchione M, Ribeiro DA, et al: Inhibition of MAPK and STAT3-SOCS3 by sakuranetin attenuated chronic allergic airway inflammation in mice. Mediators Inflamm 2019: 1356356, 2019.

31. Baus D and Pfitzner E: Specific function of STAT3, SOCS1, and SOCS3 in the regulation of proliferation and survival of classical Hodgkin lymphoma cells. Int J Cancer 118: 1404-1413, 2006.

32. Lopez-Pedrera C, Barbarroja N, Patino-Trives AM, Collantes E, Aguirre MA and Perez-Sanchez C: New biomarkers for atherothrombosis in antiphospholipid syndrome: Genomics and epigenetics approaches. Front Immunol 10: 764, 2019.

33. Johnson JL: Elucidating the contributory role of microRNA to cardiovascular diseases (a review). Vascul Pharmacol 114: 31-48, 2019.
34. Berkan O, Arslan S, Lalem T, Zhang L, Sahin NO, Aydemir EI, Korkmaz O, Egilmez HR, Cekin N and Devaux Y: Regulation of microRNAs in coronary atherosclerotic plaque. Epigenomics 11: 1387-1397, 2019.

35. Fasolo F, Di Gregoli K, Maegdefessel L and Johnson JL: Non-coding RNAs in cardiovascular cell biology and atherosclerosis. Cardiovasc Res 115: 1732-1756, 2019.

36. Pan RY, Zhao CH, Yuan JX, Zhang YJ, Jin JL, Gu MF, Mao ZY, Sun HJ, Jia QW, Ji MY, et al: Circular RNA profile in coronary artery disease. Am J Transl Res 11: 7115-7125, 2019.

37. Zhang F, Zhang R, Zhang X, Wu Y, Li X, Zhang S, Hou W, Ding Y, Tian J, Sun L and Kong X: Comprehensive analysis of circRNA expression pattern and circRNA-miRNA-mRNA network in the pathogenesis of atherosclerosis in rabbits. Aging (Albany NY) 10: 2266-2283, 2018.

38. Jiang K, Yang J, Guo S, Zhao G, Wu H and Deng G: Peripheral circulating exosome-mediated delivery of miR-155 as a novel mechanism for acute lung inflammation. Mol Ther 27: 1758-1771, 2019.

This work is licensed under a Creative Commons Attribution-NonCommercial-NoDerivatives 4.0 International (CC BY-NC-ND 4.0) License. 